\title{
Dynamic correlation effects on the plasmon dispersion in a two-dimensional electron gas
}

\author{
A. Yurtsever, ${ }^{1}$ V. Moldoveanu, ${ }^{2}$ and B. Tanatar ${ }^{1}$ \\ ${ }^{1}$ Department of Physics, Bilkent University, Bilkent, 06533 Ankara, Turkey \\ ${ }^{2}$ National Institute of Materials Physics, P.O. Box MG-7, Bucharest-Magurele, Romania
}

(Received 17 August 2002; revised manuscript received 19 November 2002; published 11 March 2003)

\begin{abstract}
The charge-density oscillations (plasmons) of a low-density two-dimensional uniform electron gas are studied within the framework of finite temperature and frequency dependent (dynamic) version of Singwi, Tosi, Land, and Sjölander theory and compared with the recent experimental results. The use of the Hartree-Fock approximation for the static structure factor leads to a finite temperature dynamical counterpart of the static Hubbard approximation. We observe important differences between dynamic and static local-field factors as well as between the corresponding plasmon dispersion laws. Our calculated plasmon energies that include dynamic correlations are in very good agreement with the recent experimental results.
\end{abstract}

DOI: 10.1103/PhysRevB.67.115308

\section{INTRODUCTION}

The dilute regime of a two-dimensional (2D) electron gas is a system of current experimental interest. This is because the technological advances in semiconductors allow very low-density samples to be prepared so that through a variety of experiments the strong correlations between the electrons may be probed. Theoretically, the uniform electron gas system with long-range Coulomb interactions has been a canonical model to study exchange and correlation effects. Thus, recent experimental efforts offer to provide a testing ground for a variety of theoretical approaches.

An important aspect of 2D electron systems is their collective excitations which are the charge-density oscillations ${ }^{1}$ (plasmons). There have been many theoretical and experimental studies devoted to their dispersion and damping properties over the years. ${ }^{2,3}$ With the advances in measurement techniques such as Raman spectroscopy plasmon dispersions in low-dimensional electronic systems become available. ${ }^{4}$

An uniform electron gas in $2 \mathrm{D}$ is characterized by a dimensionless coupling constant $r_{s}=a / a_{B}$, where $a=1 / \sqrt{n \pi}$ is the average spacing between the electrons defined in terms of the areal density $n$, and $a_{B}=\hbar^{2} \epsilon_{0} /\left(m^{*} e^{2}\right)$ is the effective Bohr radius defined in terms of the background dielectric constant $\epsilon_{0}$ and electron effective-mass $m^{*}$. Since $r_{s}$ $\sim n^{-1 / 2}$, the dilute regime of a $2 \mathrm{D}$ electron gas is considered to be a strongly interacting system. Thus, measurements of plasmon dispersion in this regime provide useful information on the correlation effects.

Recent inelastic light scattering experiments of Eriksson et $a .^{5}$ and Hirjibehedin et al. ${ }^{6}$ provide plasmon dispersion relation in ultra-low-density $2 \mathrm{D}$ electron systems going up to large wave vectors. The samples used in their experiments have densities of the order of $10^{9} \mathrm{~cm}^{-2}$, which corresponds to $r_{s} \sim 10-20$. They have found that at finite temperature the plasmon dispersion roughly follows the $\sqrt{q}$-dependence predicted by classical electrodynamics ${ }^{7}$ even in the dilute regime. However, some negative corrections from classical behavior were also observed (especially at lower temperatures) and have been associated with large correlations effects.

It is then clear that in order to understand the observed results one has to perform theoretical calculations beyond the
PACS number(s): 73.20.Mf, 05.30.Fk, 71.45.Gm

simple long-wavelength limit $(q \rightarrow 0)$, including also the correlation effects. Since real samples have a finite thickness and the measurements of Hirjibehedin et al. ${ }^{6}$ were done at finite temperature a comparison between the theory and the experimental data would be more meaningful if the former takes also into account the abovementioned effects.

A recent paper by Hwang and Das Sarma ${ }^{8}$ touches upon some of these issues and analyzes the experimental data of Eriksson et al. ${ }^{5}$ They numerically compute the plasmon dispersion using a realistic random-phase approximation (RPA), which takes into account the finite thickness, finite temperature, and static local-field corrections. The local-field corrections are described by a generalized Hubbard approximation ${ }^{9}$ for $G(q)$ that includes also the finite temperature effects. It was found by Hwang and Das Sarma ${ }^{8}$ that the finite temperature and correlation effects tend to cancel each other. This is because, the correlations reduce the strength of effective electron-electron interactions, thus lower the plasmon dispersion, whereas finite temperature increases it. However, for lower-density samples (with larger $r_{s}$ ) this cancelation takes place only if the density of the sample is adjusted by $10 \%$. It was also argued that the static Singwi, Tosi, Land, and Sjölander (STLS) theory ${ }^{10}$ gives similar results. Liu, Swierkowski, and Neilson ${ }^{11}$ noted similar cancelation effects on the plasmon dispersion in double-layer electron systems. At this point the next step forward is to tackle the problem within a theory that utilizes the dynamic local-field factor. The dynamic (or quantum) version of the STLS theory ${ }^{12}$ that improves the static correlation effects by making the localfield correction frequency dependent is a useful framework in this context. This approach is also known ${ }^{12,13}$ to produce plasmon dispersions, which always lie between the RPA result and that calculated with a static local-field factor. Dynamic local-field factors were also identified by inelastic $\mathrm{x}$-ray scattering experiments at large wave vectors in metals. ${ }^{14}$ From a theoretical viewpoint, the static local-field theories assume that the exchange-correlation hole around an electron responds rigidly to the electron motion, whereas the dynamic local-field theories attempt to modify this shortcoming. Since the relaxation time of the surrounding electrons is of the order of $1 / \omega_{\mathrm{pl}}$ (where $\omega_{\mathrm{pl}}$ is the plasmon frequency), at low densities it becomes very significant.

Motivated by these recent experiments measuring plas- 
mon dispersion in low-density 2D electron systems, and by the related theoretical problems we set out to demonstrate the importance of dynamic correlation effects in the plasmon dispersion. For this purpose we use the generalized meanfield theory to calculate the density-density correlation function from which the plasmon dispersion can be obtained. The key ingredient of this approach is the wave vector and frequency dependent local-field factor $G(q, \omega)$, which embodies the exchange and correlation effects beyond the simple RPA. In this work, we argue that the static local-field corrections are not capable of describing the observed plasmon dispersions at large $r_{s}$. Our calculations using a dynamic extension of the Hubbard approximation to $G(q, \omega)$ works very well to account for the correlation effects in recent experiments at low-density systems. The inadequacy of static local-field corrections in the context of double-layer electron systems was also pointed out by Kainth et al. ${ }^{15}$ from the analysis of their experimental results.

The rest of this paper is organized as follows. In Sec. II we introduce the formalism while Sec. III contains numerical results for plasmon dispersions and their comparison with recent experiments. We also discuss the effect of correlations in the observed plasmon energies, which include temperature and finite width contributions. Finally, we conclude with a brief summary in Sec. IV.

\section{MODEL AND THEORY}

We consider a system of homogeneous electron gas embedded in a rigid positive background in two dimensions. The electrons interact via a Coulomb potential $V(q)$ $=2 \pi e^{2} /\left(\epsilon_{0} q\right)$. Since very high mobility samples are used in the experiments ${ }^{5,6}$ we do not consider the disorder effects. In a generalized mean-field approximation the density-density correlation function of an interacting system of electrons is given by

$$
\chi(q, \omega)=\frac{\chi_{0}(q, \omega)}{1-V(q)[1-G(q, \omega)] \chi_{0}(q, \omega)},
$$

where $\chi_{0}(q, \omega)$ is the density-density response function of a noninteracting system, ${ }^{16}$ and $G(q, \omega)$ is the frequency dependent (dynamic) local-field factor. The plasmon dispersion $\omega_{\mathrm{pl}}(q)$ is readily obtained from the pole of $\chi(q, \omega)$, i.e., from the solution of

$$
1-V(q)\left[1-G\left(q, \omega_{\mathrm{pl}}\right)\right] \chi_{0}\left(q, \omega_{\mathrm{pl}}\right)=0 .
$$

The RPA is recovered if we set $G(q, \omega)=0$. In most applications, the local-field factor is approximated as frequency independent. In this static case, the plasmon energy is given by $^{12}$

$$
\omega_{\mathrm{pl}}(q) / E_{F}=\left(q / k_{F}\right)(B+1)\left(\left(q / k_{F}\right)^{2}+\frac{4}{B^{2}+2 B}\right)^{1 / 2},
$$

where $B=\left(q / k_{F}\right) /\left(\sqrt{2} r_{s}[1-G(q)]\right)$. Here $E_{F}=k_{F}^{2} / 2 m^{*}$ is the Fermi energy and $k_{F}=\sqrt{2 \pi n}$ is the Fermi wave vector (we set $\hbar=1$ ). In the case of dynamic local-field factor, the plasmon dispersion has to be obtained numerically.
We first recall some observations from the literature on the general behavior of plasmon dispersion relation $\omega_{\mathrm{pl}}(q)$ and how the correlation effects influence it. In general the correlation effects beyond the RPA lower the plasmon energy so that $\omega_{\mathrm{pl}}(q)$ calculated using local-field factors lies below that calculated within the RPA. The reason for this is that correlations effectively reduce the strength of the Coulomb interaction especially at large wave vectors. This behavior has been demonstrated in many examples. ${ }^{10,12,13}$ Another interesting fact is that when frequency dependent local-field factors are used the resulting $\omega_{\mathrm{pl}}(q)$ is typically between those calculated within the RPA and static local-field factor. Thus, in a sense we may think of $G(q, \omega)$ as giving rise to weaker correlations than its static counterpart. These observations are important because the recent experiments on very dilute 2D electron systems show that the measured values of $\omega_{\mathrm{pl}}(q)$ are not too below the RPA result. As we shall see in Sec. III it is the dynamical nature of the correlation effects that explains and fits the experimental data better.

In what concerns the static STLS approach one can use the analytical expressions for the static local-field factor of a 2D electron gas making use of exact asymptotic behaviors and accurate Monte Carlo data obtained recently by Davoudi et al. ${ }^{17}$ Their $G(q)$ may be regarded as the best available local-field factor satisfying all sum rules and limiting behavior. Qualitatively, $G(q)$ constructed by Davoudi et al. ${ }^{17}$ resembles the static STLS results at low $q$, exhibits a peak structure around $q=3.5 k_{F}$, and grows linearly at large $q$. The shape of $G(q)$ at intermediate and large $q$, however, does not affect the plasmon dispersion which is available $e^{5,6}$ only for $q \leq 1.5 k_{F}$.

We calculate the dynamic local-field factor within the framework of dynamic STLS approximation ${ }^{12}$ appropriate for a quasi-two-dimensional system. For a more realistic description of the physics involved the finite temperature effects are included both at the level of density response functions and the static structure factor $S(q)$, while the finite thickness of the sample is included by the infinite square well form factor $F(q L)$,

$$
\begin{aligned}
G(q, \omega ; T)= & -\frac{1}{n} \int \frac{d \mathbf{k}}{(2 \pi)^{2}} \frac{\chi_{0}(\mathbf{q}, \mathbf{k}, \omega ; T)}{\chi_{0}(q, \omega ; T)} \frac{V(k) F(k L)}{V(q) F(q L)} \\
& \times[S(\mathbf{q}-\mathbf{k} ; T)-1] .
\end{aligned}
$$

In this expression $\chi_{0}(\mathbf{q}, \mathbf{k}, \omega ; T)$ is the temperature dependent inhomogeneous free-electron response function to be defined below and $S(q ; T)$ is the static structure factor, which can be calculated through the fluctuation-dissipation theorem. At finite temperature $(T \neq 0)$ the static structure factor is given by: ${ }^{18}$

$$
S(q ; T)=-\frac{1}{n \pi} \int_{0}^{\infty} d \omega \operatorname{coth}\left(\frac{\omega}{2 k_{B} T}\right) \operatorname{Im} \chi(q, \omega ; T) .
$$

Using the integral representation of the Fermi function we can express the finite temperature response function in terms of zero-temperature counterpart ${ }^{19}$ 


$$
\begin{aligned}
\chi_{0}(q, u, \omega ; \mu, T)= & \int_{0}^{\infty} d \mu^{\prime} \chi_{0}\left(q, u, \omega ; \mu^{\prime}, T=0\right) \\
& \frac{1}{4 k_{B} T \cosh ^{2}\left[\left(\mu-\mu^{\prime}\right) /\left(2 k_{B} T\right)\right]} .
\end{aligned}
$$

The inhomogeneous free response function is defined as

$$
\chi_{0}(\mathbf{q}, \mathbf{k} ; \omega)=-\frac{2}{\hbar} \int \frac{d^{2} p}{(2 \pi)^{2}} \frac{f(p+\hbar q / 2)-f(p-\hbar k / 2)}{\omega-\mathbf{p} \cdot \mathbf{q} / m+i \eta},
$$

where $f(p)$ is the Fermi distribution function. More explicitly, the real and imaginary parts of the inhomogeneous free response function are given by

$$
\begin{aligned}
\chi_{0}^{\prime}(q, u, \omega)= & -\frac{k_{F}}{\pi q}\left\{u /\left(q k_{F}\right)+\operatorname{sgn}\left(\mu_{+}\right)\right. \\
& \times \theta\left(\mu_{+}^{2}-1\right)\left(\mu_{+}^{2}-1\right)^{1 / 2}+\operatorname{sgn}\left(\mu_{-}\right) \\
& \left.\times \theta\left(\mu_{-}^{2}-1\right)\left(\mu_{-}^{2}-1\right)^{1 / 2}\right\} \\
\chi_{0}^{\prime \prime}(q, u, \omega)= & -\frac{k_{F}}{\pi q}\left\{\theta\left(1-\mu_{+}^{2}\right)\left(1-\mu_{+}^{2}\right)^{1 / 2}\right. \\
& \left.-\theta\left(1-\mu_{-}^{2}\right)\left(1-\mu_{-}^{2}\right)^{1 / 2}\right\},
\end{aligned}
$$

where $u=\mathbf{q} \cdot \mathbf{k}$ and $\mu_{ \pm}=\left[ \pm \omega /\left(k_{F} q\right)-u /\left(2 k_{F} q\right)\right] \cdot \operatorname{sgn}(x)$ $=x /|x|$ and $\theta(x)$ is the unit step function. The corresponding homogeneous expressions are easily obtained taking $\mathbf{q}=\mathbf{k}$. In principle, Eqs. (1)-(5) are to be solved self-consistently within the dynamic STLS scheme. Such a calculation is laborious and beyond the scope of this work. Instead, to highlight the effects of frequency dependence we resort to a simpler approximation. We follow the derivation of the static Hubbard local-field correction $G_{H}(q)=q /\left(2 \sqrt{q^{2}+k_{F}^{2}}\right.$ within the STLS approach given by Jonson. ${ }^{20}$ Here the HartreeFock approximation $S_{0}(q ; T)$ for the static structure factor is used in evaluating Eq. (4). $S_{0}(q ; T)$ is obtained by simply replacing in Eq. (5) the free density-density response function $\chi_{0}(q, \omega ; T)$ instead of the full $\chi(q, \omega ; T)$. This allows us to obtain $G(q, \omega)$ without a self-consistent calculation, and as will be shown subsequently a good agreement with experimental results is achieved. We recall here that Hwang and Das Sarma ${ }^{8}$ have also used the Hubbard local-field factor by introducing a finite temperature generalization [i.e., replacing $k_{F}$ in the above expression for $G_{H}$ with $k_{0}(T)$ $\left.=k_{F}\left(T / T_{F}\right) \ln \left(1+e^{\mu / k_{B} T}\right)\right]$. Our proposed scheme may be regarded as the dynamical version of the static Hubbard approximation to the local-field factor, which captures the essential features of the dynamic correlation effects. Physically, the Hubbard approximation takes the depletion hole around electrons into account due to the Pauli principle.

\section{RESULTS AND DISCUSSION}

We begin this section by comparing our numerical results for $\omega_{p l}(q)$ with those obtained in the RPA and the static $G(q)$. In our calculations we use material parameters appro-

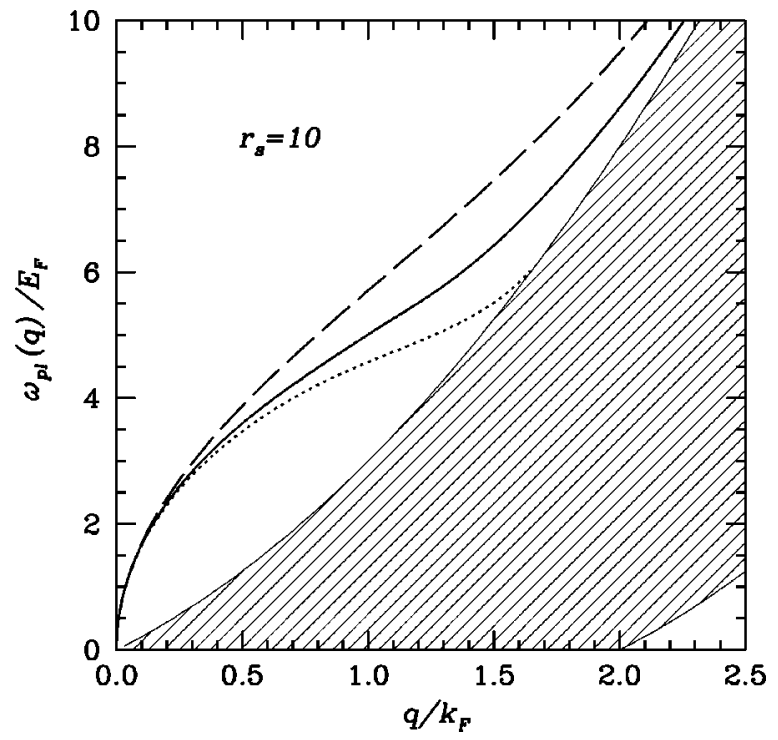

FIG. 1. Plasmon dispersions in a zero thickness 2D electron system at $r_{s}=10$ and $T=0$ as given by various theories. The dashed line indicates the result of RPA, dotted and solid lines indicate results using the static $G(q)$ and dynamic $G(q, \omega)$, respectively as explained in the text. The shaded region is the particle-hole continuum.

priate for GaAs systems. To set the stage for further discussions we show in Fig. 1 the plasmon energy $\omega_{\mathrm{pl}}(q)$ of a zero-thickness $2 \mathrm{D}$ electron system at $T=0$ calculated in the dilute regime $r_{s}=10$. The static $G(q)$ curve was obtained using the static local-field factor of Davoudi et al. ${ }^{17}$ While at small wave vectors $\left(q \leqq 0.2 k_{F}\right)$ all theories are in good agreement, at larger $q$ values (recall that the measurements of Hirjibehedin et al. ${ }^{6}$ went up to $1.6 k_{F}$ ) this is no longer true. As it is expected the RPA yields the largest plasmon frequency. $\omega_{\mathrm{pl}}(q)$ calculated using static local-field factor is far below the RPA and enters the particle-hole continuum at a critical wave-vector $q_{c}$, which is small compared to that corresponding to the dispersion law as given by dynamic STLS. Another point to be noticed is that $\omega_{\mathrm{pl}}(q)$ calculated with dynamic local-field factor always lies between the RPA result and that calculated with a static local-field factor. This is in accordance with the experimental results, which shows that $\omega_{\mathrm{pl}}$ lies not too far below the RPA plasmon dispersion curve.

To understand the differences between the dynamic and static local-field factors within the STLS we have also compared the corresponding local-field factors in Fig. 2. It turns out that the inclusion of the frequency dependence in the STLS theory (calculated here in the dynamic Hubbard approximation) cause significant changes in the local-field factor. We first note that at finite wave-vectors there is a big difference even between the purely static $G(q)$ (as calculated by Davoudi et al. $)^{17}$ and $G(q, 0)$, i.e., when the frequency is set equal to zero. Second, the $q$ dependence of $G(q, \omega)$ for finite frequencies is similar to that of $G(q, 0)$. Only for large frequencies $(\omega \rightarrow \infty)$ the dynamic local-field factor approaches the static $G(q)$.

We now compare our calculation of the plasmon disper- 


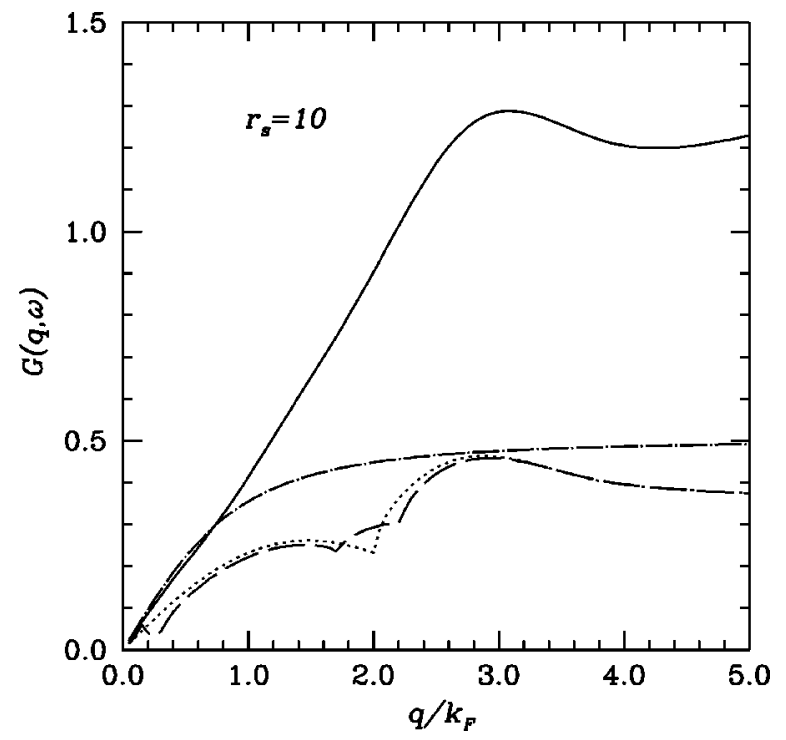

FIG. 2. The wave-vector dependence of dynamic and static local-field corrections at $r_{s}=10$. Thick solid line- $G(q)$, dotted line- $G(q, 0)$, dash dotted line-Hubbard approximation, dashed line- $G\left(q, \omega=0.5 E_{F}\right)$.

sion curves with the experimental results of Hirjibehedin et $a l .{ }^{6}$ To make the comparisons more appropriate, the results were obtained for $L=330 \AA$, which is close to the experimental samples and the temperatures effects are fully incorporated. We use the experimentally quoted $2 \mathrm{D}$ electron densities, although a certain (10\%) margin of uncertainty exists in their determination. The temperatures are also given in terms of the Fermi temperature $T_{F}$ of the corresponding sample. Note that since $T$ is of the same order or larger than $T_{F}$ the temperature effects cannot be neglected. Figure 3 shows $\omega_{\mathrm{pl}}(q)$ as a function of the wave vector at $r_{s}=8.7$ and $r_{s}=19.7$, which correspond to the highest- and lowestdensity samples used in experiment. ${ }^{6}$ The experimental data of Hirjibehedin et al. ${ }^{6}$ are indicated by solid dots. Results of our calculations using dynamic local-field factors are given by the solid lines. We observe that a good agreement exists between our calculations and the experimental data. For comparison the finite temperature and quantum-well width corrected RPA and static local-field correction $G(q)$ results are also plotted. We use the finite temperature and finite width corrected static Hubbard approximation to calculate $G(q ; T)$ and subsequently the plasmon dispersion $\omega_{\mathrm{pl}}(q)$. The discrepancy between the theoretical curves from RPA and $G(q, \omega)$ for large $q$ values increases with increasing $r_{s}$ showing thus how important the role of the correlation effects are. Another important observation here is that the static Hubbard approximation leads to $\omega_{\mathrm{pl}}(q)$ lying below the experimental data. This demonstrates once more the failure of the static approach to give an accurate estimate of the correlation effects in the large $q$ region of the plasmon dispersion in low-density electron systems.

We extend the comparison with the experiment further in Fig. 4, where the data taken from Fig. 3 of Ref. 6 are shown along with our calculations for a dilute sample $\left(r_{s}=15.2\right)$ at different temperatures. The reported temperatures $T$
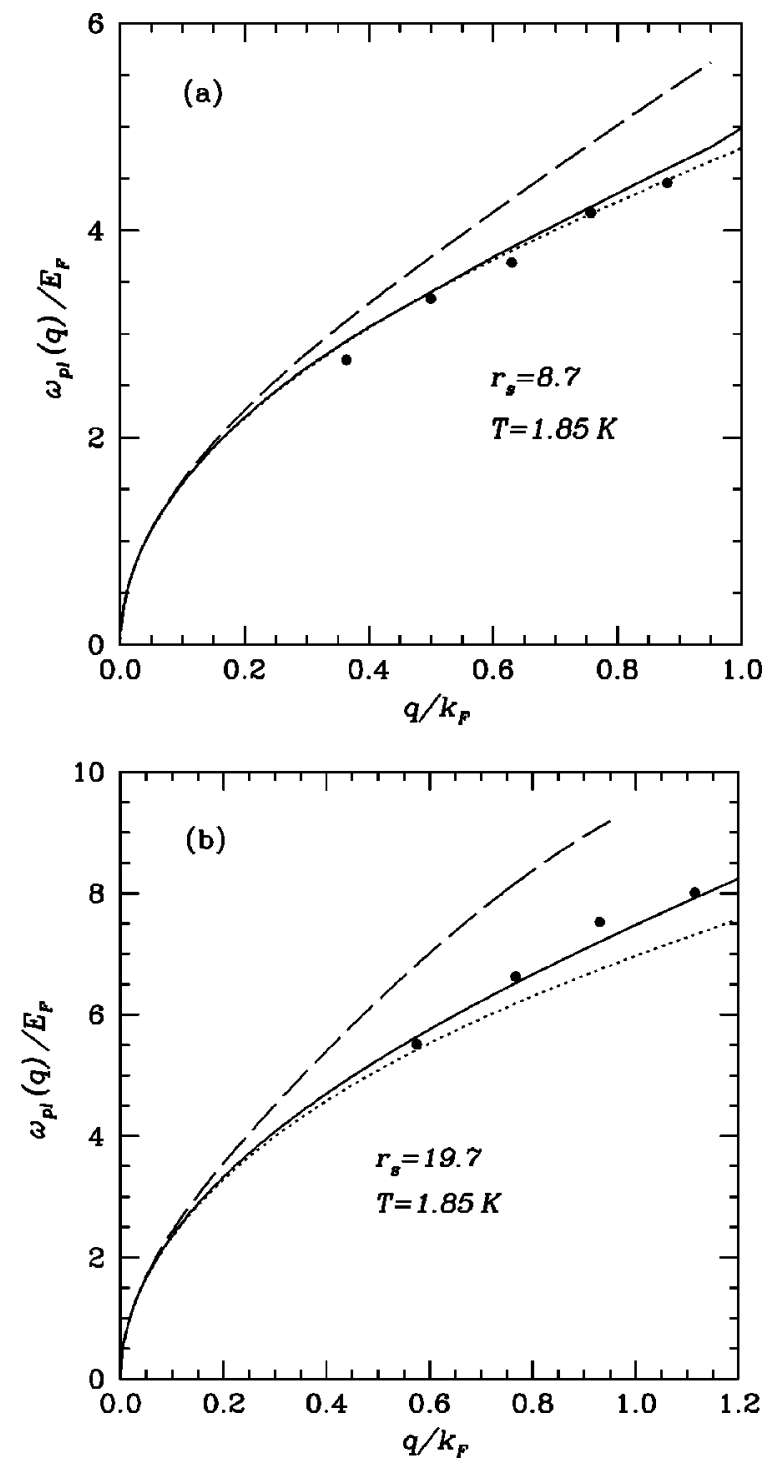

FIG. 3. Plasmon dispersion of the dilute $2 \mathrm{D}$ electron gas for different $r_{s}(L=330 \AA)$. (a) $r_{s}=8.7, T=1.85 \mathrm{~K} \approx 1.1 T_{F}$, (b) $r_{s}$ $=19.7, T=1.85 \mathrm{~K} \approx 5.7 T_{F}$. RPA results are plotted with dashed lines and the experimental data of Ref. 6 with solid circles. Dotted lines indicate $\omega_{\mathrm{pl}}(q)$ using the static Hubbard approximation.

$=0.25 \mathrm{~K}$ and $T=1.85 \mathrm{~K}$ correspond to $0.46 T_{F}$ and $3.4 T_{F}$, respectively, for the $r_{s}=15.2$ sample, where $T_{F}$ is the Fermi temperature. Under these conditions, the temperature has a non-negligible impact on the plasmon dispersion. We obtain a similar level of agreement (not shown) at a larger temperature $T=4.55 \mathrm{~K}\left(=8.3 T_{F}\right)$, where the plasmon dispersion is largely determined by the temperature effects.

Finally in Fig. 5 one can distinguish the finite thickness correction from the thermal effects on the plasmon dispersion. The dotted line contains only correlation effects $(T$ $=0, L=0)$. The dashed line $(T=0, L=330 \AA)$ shows the expected decrease in $\omega_{\mathrm{pl}}(q)$ due to the finite thickness. Important remarks are to be traced from the full line that contains all corrections: finite temperature, finite thickness, and dynamical correlation effects. First, the temperature has a larger effect on the plasmon dispersion than the finite thick- 


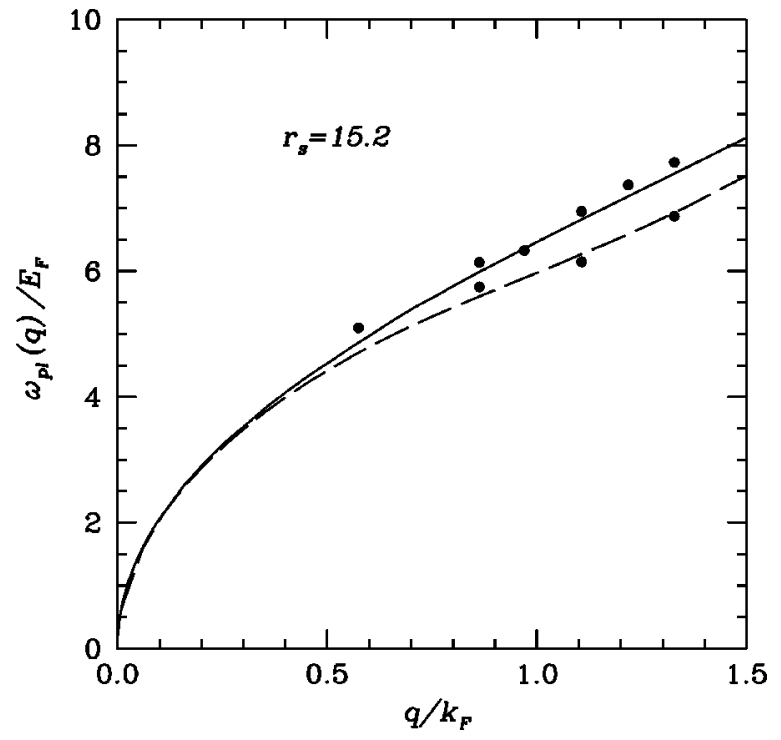

FIG. 4. Temperature dependence of the plasmon dispersion $\left(r_{s}\right.$ $=15.2$ and $L=330 \AA$ ). Dashed line corresponds to $T=0.25 \mathrm{~K}$ $\approx 0.458 T_{F}$ and the full line to $T=1.85 \mathrm{~K} \approx 3.39 T_{F}$. Experimental points of Ref. 6 are marked with solid circles.

ness (for the specific values chosen here) and second, within our formalism all the above mentioned effects contributes to $\omega_{\mathrm{pl}}(q)$ in such a way that the theoretical curve is in a good agreement with the reported measurements. Having in mind the comparison made in Fig. 1 and the fact that for dilute samples the static local-field corrections was found to be too strong to be completely canceled with the thermal effects ${ }^{8}$ we are led to the conclusion that the good compensation of the dynamical correlation effects is due to the fact that they yield plasmon dispersion curve lying above that calculated using static correlations.

From a theoretical standpoint the determination of exact or physically relevant dynamic local-field factor $G(q, \omega)$ is a subject of interest. Starting from the early work of Czachor et $a l,{ }^{21}$ there has been numerous attempts at calculating $G(q, \omega)$ for $2 \mathrm{D}$ electron-gas systems. ${ }^{22}$ As mentioned before there are indications in the experimental data for plasmon dispersion and damping properties which put into evidence the importance of dynamic correlation effects. The good agreement we obtain using a simple version of $G(q, \omega)$, namely, the dynamic version of the Hubbard approximation, is somewhat surprising. We surmise that it should work better than the fully self-consistent calculation within the dynamic STLS. Our results show the importance of dynamic correlation effects in low-density electron systems, but the amount of such effects embodied in $G(q, \omega)$ appears to rather delicately depend on the level of approximation being used. Further theoretical work is necessary for clarification of the exact nature of $G(q, \omega)$. A different approach to dynamic correlation effects was taken by Neilson et al., ${ }^{23}$ where they employed Mori formalism to obtain the relaxation function. However, their calculated plasmon dispersions are slightly lower than those with static correlations,

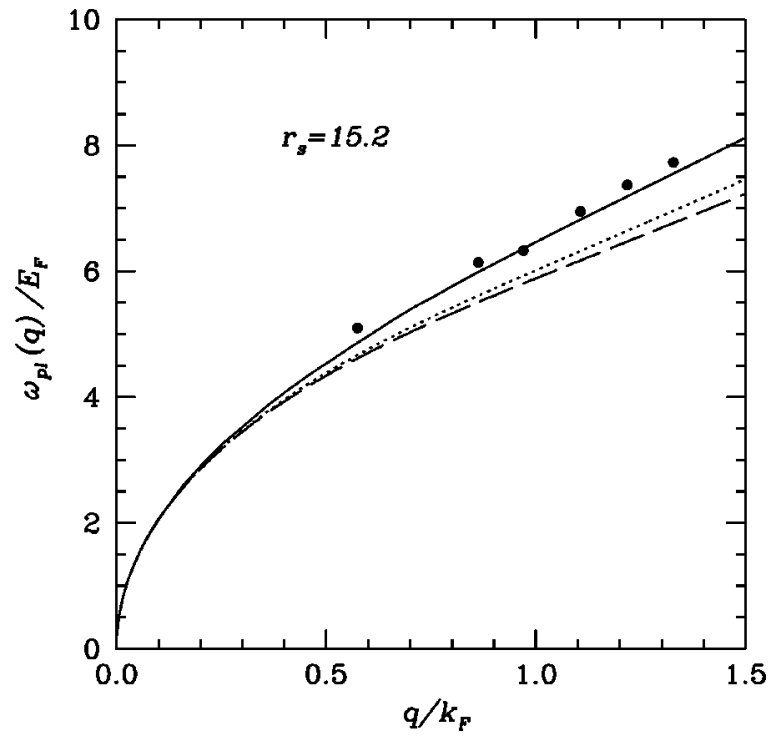

FIG. 5. Finite thickness and finite temperature contributions to the plasmon dispersion at $r_{s}=15.2, L=330 \AA$, and $T=1.85 \mathrm{~K}$ (solid line) along with the experimental data of Ref. 6 (solid circles). Finite thickness, zero-temperature (dashed line) and zerothickness, zero-temperature (dotted line) dispersions are also drawn for comparison.

which would make the agreement with Hirjibehedin et al. ${ }^{6}$ data less satisfactory.

\section{SUMMARY}

In this work we have considered the plasmon dispersion relation in a dilute $2 \mathrm{D}$ electron gas. Motivated by the recent experiments on such systems we have found that the observed results are best understood in terms of dynamic correlation effects. The key ingredient of our approach is the wave vector and frequency dependent local-field factor $G(q, \omega)$, which embodies the exchange and correlation effects beyond the simple RPA. We have demonstrated the inadequacy of purely static local-field factors in reproducing the observed plasmon dispersions. Our calculations show that a straightforward extension of the Hubbard approximation, which now includes frequency and temperature dependence, can explain the experimentally observed plasmon dispersions at very low-density samples. Further experiments at low density and temperatures and in the range of large- $q$ values would be useful to assess the role of exchangecorrelation effects. Theoretical work should concentrate on the systematic understanding of dynamical correlation effects.

\section{ACKNOWLEDGMENTS}

This work was partially supported by the Scientific and Technical Research Council of Turkey (TUBITAK) under Grant No. TBAG-2005, by NATO under Grant No. SfP971970, by the Turkish Department of Defense under Grant No. KOBRA-001, and by the Turkish Academy of Sciences (TUBA). V. M. acknowledges support from NATOTUBITAK. 
${ }^{1}$ D. Pines and P. Nozieres, The Theory of Quantum Liquids (Addison-Wesley, New York, 1989).

${ }^{2}$ T. Ando, A.B. Fowler, and F. Stern, Rev. Mod. Phys. 54, 437 (1982).

${ }^{3}$ For a recent comprehensive review see, M.S. Kushwaha, Surf. Sci. Rep. 41, 5 (2001).

${ }^{4}$ A. Pinczuk and G. Abstreiter, Light Scattering in Solids V, edited by M. Cardona and G. Güntherodt (Springer, Heidelberg, 1989), p. 153.

${ }^{5}$ M.A. Eriksson, A. Pinczuk, B.S. Dennis, C.F. Hirjibehedin, S.H. Simon, L.N. Pfeiffer, and K.W. West, Physica E 5, 165 (2000).

${ }^{6}$ C.F. Hirjibehedin, A. Pinczuk, B.S. Dennis, L.N. Pfeiffer, and K.W. West, Phys. Rev. B 65, 161309 (2002).

${ }^{7}$ A.L. Fetter, Phys. Rev. B 10, 3739 (1974); P.M. Platzman and N. Tzoar, ibid. 13, 3197 (1976).

${ }^{8}$ E.H. Hwang and S. Das Sarma, Phys. Rev. B 64, 165409 (2001).

${ }^{9}$ J. Hubbard, Proc. R. Soc. London, Ser. A 243, 338 (1957).

${ }^{10}$ K.S. Singwi, M.P. Tosi, R.H. Land, and A. Sjölander, Phys. Rev. 176, 589 (1968); K.S. Singwi and M.P. Tosi, Solid State Phys. 36, 177 (1981).

${ }^{11}$ L. Liu, L. Świerkowski, and D. Neilson, Physica B 251, 937 (1998).
${ }^{12}$ R.K. Moudgil, P.K. Ahluwalia, and K.N. Pathak, Phys. Rev. B 52, 11945 (1995).

${ }^{13}$ C. Bulutay and B. Tanatar, Europhys. Lett. 43, 572 (1998); B. Tanatar and C. Bulutay, Phys. Rev. B 59, 15019 (1999).

${ }^{14}$ B.C. Larson, J.Z. Tischler, E.D. Isaacs, P. Zschack, A. Fleszar, and A.G. Eguiluz, Phys. Rev. Lett. 77, 1346 (1996).

${ }^{15}$ D.S. Kainth, D. Richards, H.P. Hughes, M.Y. Simmons, and D.A. Ritchie, J. Phys.: Condens. Matter 12, 439 (2000).

${ }^{16}$ F. Stern, Phys. Rev. Lett. 18, 546 (1967).

${ }^{17}$ B. Davoudi, M. Polini, G.F. Giuliani, and M.P. Tosi, Phys. Rev. B 64, 153101 (2001).

${ }^{18}$ H.K. Schweng and H.M. Böhm, Phys. Rev. B 48, 2037 (1993).

${ }^{19}$ P.F. Maldague, Surf. Sci. 73, 296 (1978).

${ }^{20}$ M. Jonson, J. Phys. C 9, 3055 (1976).

${ }^{21}$ A. Czachor, A. Holas, S.R. Sharma, and K.S. Singwi, Phys. Rev. B 25, 2144 (1982).

${ }^{22}$ Z.X. Qian and G. Vignale, Phys. Rev. B 65, 235121 (2002); H.J. Schulze, P. Schuck, and N. Van Giai, ibid. 61, 8026 (2000); K. Takayanagi and E. Lipparini, ibid. 52, 1738 (1995) and references therein.

${ }^{23}$ D. Neilson, L. Świerkowski, A. Sjölander, and J. Szymanski, Phys. Rev. B 44, 6291 (1991). 